\title{
LES « ÉCLATS DU MONDE » DANS LA FIGURE DU NARRATEUR ET LA « PAROLE DE LIBERTÉ » DANS VEILLES ET MERVEILLES CRÉOLES. CONTES DU PAYS Martinique de PATRick Chamoiseau
}

\author{
Milena FuČÍKOVÁ \\ Université Charles, Prague
}

\begin{abstract}
En): The paper deals with a specific interpretation of literary narrative in Chamoiseau's Veilles et Merveilles créoles. We focus on the analysis of lyrical forms in narrative texts, in particular, on how the personification of the narrator introduces an autonomous representation of the author and, more importantly, the personification of a discourse which seeks to explore images of movement as a metaphor for reflection on his narrative. Patrick Chamoiseau's images of metamorphosis represent the basis for his own poetics.
\end{abstract}

Keywords (En): Patrick Chamoiseau ; narrator ; Creoleness ; orality ; true memory ; metaphor

Mots-clés (Fr): Patrick Chamoiseau ; la figure du narrateur ; creolité ; oral ; mémoire vraie ; métaphore

\section{Introduction}

Dans l'œuvre romanesque de Patrick Chamoiseau, le narrateur apparaît comme une figure symbolique, comme un thème récurrent, et comme une variation «en toute époque ». Cette figure semble inséparablement liée aux problématiques que l'auteur martiniquais explore au fil de tous ses romans. Les grands textes de ce chantre de la créolité, de Chronique des sept misères, qui date de 1988, à La matière de l'absence, publié en 2016, sont sans aucun doute marqués par trois notions emblématiques. Premièrement, il s'agit de la créolité qui représente un concept tout aussi intéressant que théorique de l'identité créole en tant que mosaïque composée de différentes populations du monde, proposée dans le manifeste L'Eloge de la créolité par Bernabé, Confiant et par Chamoiseau luimême en 1989'. Deuxièmement, il y a la notion de l'oralité et celle, bien sûr, de la littérature orale, trait prééminent en Martinique ${ }^{2}$. Troisièmement, il y a l'idée de l'opacité développée notamment par Édouard Glissant afin de faire non seulement le pari de la diversité heureuse mais surtout d'exprimer le fait qu'une transparence totale, logique et claire est simplement impossible dans l'esthétique et dans la langue de l'autre.

Patrick Chamoiseau a mis en pratique ces trois concepts théoriques en inventant un mode de narrativité original, fondé tout à la fois sur l'héritage du conteur, sur l'autofiction et sur son imaginaire poétique particulier. Outre le

1 Comparez «Ni Européens, ni Africains, ni Asiatiques, nous nous proclamons Créoles.» (Bernabe ; Chamoiseau ; Confiant, 1993 : 13).

${ }^{2}$ Au début des années 1990, de multiples polémiques en France surgirent à propos de l'oral et de l'écrit en littérature martiniquaise. Comparez p.e. la formulation d'Edouard GLISSANT : " cette lisière de l'oral et de l'écrit où se joue une des perspectives actuelles de la littérature » $(1988: 6)$. 
modèle de narration dynamique, l'écriture de Patrick Chamoiseau joue pleinement avec un narrateur humoristique et actif, qui selon KUNDERA aurait existé «de Rabelais à Laurence Sterne» (2009 : 99). En m'appuyant sur les contes créoles du recueil de l'auteur, intitulé Veilles et Merveilles Créoles. Contes du pays Martinique publié en 2013, je me propose d'examiner tout d'abord certains des traits de la figure du narrateur chamoisien, de montrer ses façons d'être ou de ne pas être dans le récit, et ensuite les jeux, ou bien les enjeux d'un tel fonctionnement. Pour finir, je voudrais souligner la relation au monde de ce narrateur cachotier à partir de l'image de la traversée en bateau ainsi que son désir de métamorphose.

Tout d'abord, cet auteur contemporain écrit en français tout en se plaçant dans un contexte de multilinguisme. Patrick Chamoiseau est né en 1953 à Fort-deFrance en Martinique. Il est parfois désigné comme "francophone » ${ }^{3}$, car il pratique une écriture entre le français et une autre langue qui est sa langue maternelle, et il serait de ce fait « condamné à penser la langue » (GAUVIN, 1997 : 8). C'est bien entendu le créole. À ce jour, Patrick Chamoiseau a publié une dizaine de romans, mais également des essais, des manifestes, du théâtre conté et des contes. Le prix Goncourt de 1992, pour son troisième roman Texaco, lui a apporté une reconnaissance plus large du public. Ses trois derniers romans, à savoir Les Neufs Consciences du Malfini, publié en 2009, L'Empreinte à Crusoé, publié en 2012, et enfin La Matière de l'Absence, publié en 2016, sont des romans à la limite des poèmes en prose aux résonances écologiques pour Malfini, à la réflexion philosophique des Lumières pour Crusoé jusqu'à une évocation hyperbolique de la figure maternelle dans La Matière de l'Absence. Cet auteur soulève des questions importantes de l'Histoire, de la mémoire, et bien sûr du passé colonial français. Dans ses romans, il tente de «nommer l'innommable». Ces thèmes-phares s'entremêlent dans une poétique qui puise une part de son inspiration dans la poésie et la musique moderne, notamment dans le jazz. Il aborde aussi les thèmes de la dissimulation et de l'opacité qui nous intéressent plus particulièrement ici.

\section{L'héritage du conteur}

Dans la lignée d'un Rabelais, d'un Diderot ou d'un Sterne, chez Chamoiseau, on sait toujours qui raconte, ou qui est l'instance narrative selon la terminologie de Franz K. Stanzel. En général, à l'instar du conteur, le narrateur évoque ses personnages comme on parlerait d'un ami, et il indique toujours le sens et la moralité des récits qu'il propose à ses lecteurs. Il s'adresse directement aux autres

\footnotetext{
${ }^{3}$ Le terme «écrivain francophone» est problématique car il renvoie à la base à des repères politiques. La notion de la «francophonie » réfère dans son acceptation la plus large à l'ensemble des pays qui ont en usage la langue française, mais l'espace de ce qu'on appelle la «Francophonie littéraire » ne couvre pas exactement le même champs d'extension. Dans les Voleurs de la langue, Jean-Louis JOUBERT emploie cette métaphore de «voleurs de langue » l'ensemble des auteurs qui ont osé l'aventure d'écrire en français afin d'être récompensés par le pouvoir de dire «l'indicible dans la langue d'origine » $(2006: 132)$.
} 
(à ses personnages, et à ses lecteurs), tout en se gardant bien d'en dire de trop sur sa propre personnalité.

Dans la préface aux Veilles et Merveilles Créoles, le conteur apparaît d'emblée dans un rôle double :

Au bas du morne, dans le quartier des esclaves, un personnage émerge de l'une des cases à nègres. Des esclaves sont là, sous un vieil arbre, qui l'attendent, qui l'espèrent. Cet homme n'a pourtant rien de particulier; d'âge mûr, il n'est ni plus ni moins insignifiant que les autres. Le jour, il n'est qu'un nègre de cannes qui travaille, souffre, transpire, et qui vit dans la crainte, la révolte ravalée. (CHAMOISEAU, $2013: 7$ )

Le conteur selon Chamoiseau ressemble parfaitement aux autres, il n'a donc rien de plus ni de moins que les autres esclaves, lui aussi, il a peur, lui aussi, il a faim, lui aussi, il est révolté. Or, il a une qualité en plus, une qualité essentielle : il dissimule plus que les autres, car il est une sorte de modèle, de figure emblématique, bref une autorité à la fois visible et invisible : "Peut-être même est-il plus discret que plus d'un » (CHAMOISEAU, $2013: 7$ ).

Pendant le jour il se tait, afin de montrer sa force la nuit : « Mais la nuit, une exigence obscure dissipe sa lassitude, le dresse, l'habite d'une force nocturne et quasi clandestine : celle de la Parole dont il devient le Maître. C'est le Conteur » (Chamoiseau, $2013: 8$ ).

Dans la plupart des romans de Chamoiseau, le personnage du conteur reste rare, sauf dans Solibo Magnifique, bien sûr, mais même dans ce récit initiatique c'est justement lui, le dernier véritable conteur, qui meurt dès la première page ${ }^{4}$. Nombreuses sont cependant les allusions au conteur ainsi qu'au message qu'il véhicule. Ce personnage est à la fois présent et diffus. Il est absent et présent à l'instar d'un personnage mythique. Lié à d'autres, il revêt des fonctions diverses. Dans Esclave vieil homme et le molosse notamment, le conteur est associé, comme toujours chez Chamoiseau, à la liberté : «Le Papa-conteur de l'Habitation était un bougre assez insignifiant. Il aspirait la vie autour de lui pour sustenter son verbe. Et de ce verbe, il éveillait la vie. Il parolait et faisait rire. Et le rire déployait les poitrines, les amplifiait. Les haines, les désirs, les cris perdus et les silences de tous s'exprimaient par sa bouche » (CHAMOISEAU, $1997: 47$ ).

La stratégie de ce personnage-clé demeure apparemment simple. Il se montre tout d'abord soumis, réduit au silence, mais ensuite, dès qu'il se met à parler, il devient puissant : c'est donc grâce à la parole qu'il prend le pouvoir. Dans Solibo Magnifique, cette égalité entre le conteur et les autres personnages est particulièrement marquante. Patrick Chamoiseau, dissimulé dans le récit dans la peau d'un personnage fictif, nommé de la même manière que lui - «Ti-Cham », « Oiseau de Cham », «Chamzibié » ou encore «marqueur de paroles »-dialogue avec le conteur Solibo Magnifique. Ensemble, ils évoquent avec brio les différences entre la littérature orale et la littérature écrite, et d'emblée, ils

\footnotetext{
${ }^{4}$ Comparez : «Au cours d'une soirée de carnaval à Fort-de-France, entre dimanche Gras et mercredi des Cendres, le conteur Solibo Magnifique mourut d'une égorgette de la parole, en s'écriant : Patat'sa !... »(ChAmoiseau, $1988: 25)$.
} 
s'interrogent sur la place de l'auteur dans le texte, dissimulé dans la figure du narrateur :

Solibo Magnifique me disait : «...Oiseau de Cham, tu écris. Bon. Moi, Solibo, je parle. Tu vois la distance? Dans ton livre sur Manman Dlo, tu veux capturer la parole à l'écriture, je vois le rythme que tu veux donner, comment tu veux serrer les mots pour qu'ils sonnent à la langue. Tu me dis : Est-ce que j'ai raison, Papa ? Moi, je dis : On n'écrit jamais la parole, mais des mots, tu aurais dû parler. Ecrire, c'est comme sortir le lambi de la mer pour dire : voici le lambi ! La parole répond : où est la mer? Mais l'essentiel n'est pas là. Je pars, mais toi tu restes. Je parlais, mais écris en annonçant que tu viens de la parole. Tu me donnes la main par-dessus la distance. C'est bien, mais tu touches la distance... ». (CHAmoiseau, 1988 : 52-53)

\section{De la dissimulation du narrateur à un imaginaire poétique particulier}

Le conteur créole tout comme le narrateur chamoisien sont là pour retracer le passé violent de leur peuple, né de l'esclavage. Ils souhaitent dire tout ce qui n'a pas été dit, tout ce qui a été caché, tout ce qui n'a pas pu avoir lieu d'être : des vides, des traumatismes, des blancs de la grande Histoire écrite. La force du verbe, le pouvoir du dire sont des thèmes évoqués dans plusieurs des contes et apparaissent ainsi, de manière récurrente, pratiquement dans tous les textes de Patrick Chamoiseau. Toujours dans la même préface, l'auteur évoque la situation paradoxale qui le pousse à dissimuler son message car grâce à cette dissimulation, il pourra mieux l'exprimer :

[...] le maitre sait que (le Conteur créole) parle, le maître tolère qu'il parle, parfois même le maître entend ce qu'il dit; sa Parole se doit donc d'être opaque, détournée, d'une signifiance diffractée en mille miettes sibyllines. Sa narration tournoie sur de longues digressions humoristiques, érotiques, souvent même ésotériques. Son dialogue avec l'auditoire est incessant, ponctué d'onomatopées et de bruitages, qui visent autant à retenir l'attention qu'à ôter de son propos toute évidence alors dangereuse. (CHAMOISEAU, $2013: 8-9$ )

Une fois que l'histoire est dite ou que le récit dans un roman est terminé, le narrateur chez Chamoiseau se précipite pour tout tourner en dérision, il montre qu'il n'a pas d'importance, et surtout qu'il y a une limite très nette entre lui et ses personnages. Dans La Madame Kéléman, on peut lire les propos suivants : « La petite fille, dit-on, vécut bien bien-contente avec le grand serpent devenu bon garçon. Sa case est tellement ouverte à tous que même les vagabonds comme moi y passent après les contes pour s'arroser la soif et chanter sa beauté. » (Chamoiseau, $2013: 25$ )

Ou bien, dans un autre conte, intitulé Glan-Glan, l'oiseau craché, on apprend dans la première phrase que le narrateur aime bien rester à la maison le Vendredi saint, à la différence de ses personnages qui viennent de vivre ce jour-là une mésaventure car ils sont sortis pour chasser un oiseau magnifique. Ainsi, le narrateur se distingue de ses personnages tout de suite dès le début du conte : « Moi, je reste au sage chez moi à manger des beignets et à boire de l'eau pure. » (Chamoiseau, 2013: 25)

Dans la dernière phrase aussi, il se fait tout petit, insignifiant, comme si de rien n'était : «L'oiseau refit ses comptes, et s'envola comme s'envolent les rêves. On 
dit qu'après cette histoire, la mauvaise femme devint aimable et douce, et même végétarienne, si bien que le pauvre homme ennuyé de ce bonheur en épousa une autre. Mais ça, c'est son affaire ! Moi, le Vendredi saint je reste au sage chez moi... » (CHAMOISEAU, 2013 : 58)

À la fin du conte nommé Accra de la richesse, au contraire, on sait que si le narrateur n'est pas directement son personnage rusé, du moins, il approuve ses actes et sort pour une fois de sa cachette rhétorique. Ici, il s'agit de Ti-zèb, qui grâce à sa ruse vient d'acquérir une grande maison et alors qu'à la base, il était très mal parti dans la vie, juste avec un beignet durci de sa maman qui est morte. Le narrateur approuve ses actes en disant : «En tout cas, à l'heure des voyages, j'ai toujours un accra bien au fond de ma poche. » (CHAMOISEAU, 2013 : 71)

Chez Chamoiseau, la question de la langue est centrale. René Depestre aimait à dire qu'il était bon et juste de « conduire la langue française à la rivière et de lui frotter le corps avec des herbes parfumées ». Pour Patrick Chamoiseau, la langue française demeure l'outil privilégié qui permet de nommer, ou de donner le nom à l'innommable. Même le perroquet de Robinson, personnage dans Empreinte à Crusoé qui dit «pauvre Robinson, où es-tu ? » évoque cette question essentielle de langage. Ainsi, Edouard Glissant a raison de souligner que le projet du conteur créole, et de la même façon du narrateur chamoisien, c'est «presque d'obscurcir en révélant » (GLISSANT cité par CHAMOISEAU, 2013: 9). De former et d'informer dans l'hypnose de la voix ou du mystère du verbe. De se cacher, de se montrer pour dire, et puis de disparaître à nouveau.

Le narrateur s'exprime dans un style original, étonnant, et surtout poétique. Bien souvent, il est question du passé esclavagiste, du bateau négrier, de la cale, qui représente à mon sens, un véritable topos des récits chamoisiens. Ernst Robert Curtius évoque la traversée sur un navire comme une image essentielle de l'imaginaire de la littérature occidentale ${ }^{5}$, car on la retrouve chez Dante ou chez Arioste. Nous nous souvenons de la nef des fous d'Erasme Rotterdam, nous connaissons le «Le Bateau ivre» d'Arthur Rimbaud, le symbole même de la poésie moderne. Rimbaud, le poète, à qui Chamoiseau doit beaucoup tout comme aux poètes surréalistes tels que Aimé Césaire notamment, a écrit son fameux «je suis nègre » sans pour autant jamais avoir été négrier et déclare dans ce texte qu'il s'est baigné « dans le Poème de la Mer », ce qui représente, contre toute attente, une image tout à fait traditionnelle de la poésie occidentale. Le poète tel un bateau ivre fait la traversée pour atteindre la côte d'un monde rêvé, d'un monde imaginaire, d'un monde fantastique de la poésie et de l'enfance. En revanche, et de manière logique, pour Chamoiseau, le bateau, c'est le bateau négrier : c'est toujours la cale, c'est le trajet de la Traite du $17 \mathrm{e}$ et du $18 \mathrm{e}$ siècle, ce triangle infernal entre la côte sénégalaise, les Antilles françaises et les ports de Nantes et de Saint-Malo. Somme toute, il s'agit de l'indicible, l'innommable. C'est le monde sensé, le «monde ancien » comme dirait Apollinaire, qui vole en éclats, le monde compréhensible et intelligible qui explose, qui perd tout de son sens. De ce fait, Edouard Glissant propose le détour pour exprimer une part de ce qui est

\footnotetext{
${ }^{5}$ Comparez l'opus de CurTiUs, Ernst Robert (1998), Evropská literatura a latinský středověk. Praha, Triáda, pp. 144-148.
} 
indicible, un détour, car justement la dissimulation narrative, c'est un détour symbolique, voire «emblématique»: «un système de contre-valeurs ou de contre-culture, où se manifeste en même temps une impuissance à se libérer globalement et un acharnement à tenter de le faire » (CHAMOISEAU, $2013: 8$ ).

\section{Nommer de manière détournée : par une image métaphorique}

Chamoiseau crée son propre langage parsemé de mots étranges et d'expressions opaques, inconnues dans la belle langue française qui produisent d'un côté une magie souterraine, une séduction du récit et de l'autre côté une distance, ou plutôt une note d'humour.

Dans l'Esclave vieil homme et le molosse, Patrick Chamoiseau remonte très loin dans l'histoire martiniquaise. Il explore une scène «archétypale » qui hante jusqu'à nos jours l'imaginaire créole. Un vieil esclave, silencieux et insignifiant, une sorte de double du conteur, s'échappe d'une plantation martiniquaise et son Maître envoie un chien à ses trousses. Le thème du bateau est suggéré surtout par les images, et cela de manière minimaliste. Le personnage de l'esclave n'a pas d'enfants, mais il a le sentiment d'habiter les îles depuis toujours. Parfois, l'esclave se rend au port pour observer les bateaux négriers qui arrivent :

Parfois, ces navires entraient dans le port en une ivresse errante. On découvrait alors leur cargaison ferrée, asséchée par la faim et les fièvres à frappe jaune. L'entrepont et les haubans étaient déserts. Les voiles orphelines devenaient de grandes feuilles assoiffées, et les filins se dénouaient à dire des cordes de pendus. L'équipage avait sombré dans la boucle d'un mystère. Les tonneaux d'huile, de viande salée ou d'eau potable grouillaient des mêmes vers, lesquels semblaient attendre (ou annoncer) une fin des temps. De chaque goupille du pont, de petites flammes naissaient pour s'évanouir là même dans un relent de basilic. Nul ne pouvait acquérir ces épaves enchaînées que l'on sortait des soutes. Sans même les nourrir, le Gouverneur affrétait un vapeur militaire pour les acheminer en quelque coin sans retour de la côte du Brésil. (CHAMOISEAU, 1997 : 36-37)

Le bateau négrier est ici assimilé à un bateau ivre. Le langage poétique permet d'invalider d'entrée de jeu les catégories du passé, du présent et du futur. Plus tard, lorsque l'esclave court dans les bois, il retrouve des parts de sa mémoire. Sous forme d'hallucinations, sous forme d'images chaotiques : la traversée de l'Atlantique, la violence négrière et esclavagiste se présentent à son esprit de manière indirecte, dissimulée toujours, mais surtout métaphorique. Le langage poétique personnel basé essentiellement sur les figures d'analogie permet de remplacer et bien souvent d'adoucir la cruauté de la pure connaissance scientifique. L'auteur intervient à la fin du récit pour prendre la parole et évoquer son langage : «Un langage de conte et de souffle de course. Un langage qui dirait sa parole en la signalant muette. Un langage qui mélangerait le silence de sa langue aux frappes dominatrices qui écraserait son dire. (CHAMOISEAU, 1997 : 145). 


\section{Conclusion}

S'il est faux de parler de symboles à propos de Chamoiseau, il convient en revanche d'insister sur les images fondamentales comme celle du bateau (avec ses variantes : vaisseau fantôme dans le conte La personne qui asséchait les cœurs), point d'appui au milieu du déluge pour une possible genèse ou re-genèse.

La voie est ouverte aussi aux linguistes et aux stylisticiens pour étudier les métamorphoses de la phrase chamoisienne, d'abord traditionnelle, ancrée dans l'imaginaire créole, puis devenant de plus en plus fluide et épurée jusqu'à parfois une incompréhensible poétique : le procédé métaphorique se construit chez Chamoiseau fréquemment dans l'espace infime entre le français et le créole (il s'agit sans aucun doute d'une sorte de dissimulation, car le créole reste la langue muette, mais tout aussi bien d'un nouvel imaginaire poétique) procédé caractéristique de l'ambiguïté qui marque toute la démarche de Chamoiseau.

Sans négliger la part dissimulée de sa poétique, le lecteur peut être sensible au caractère libérateur de ses meilleurs romans. Lire Chamoiseau, c'est comme s'ouvrir à un langage nouveau. L'écriture apparaît comme une expérience humaine, une évolution imaginaire au bord d'un bateau où on a à jeter par-dessus bord toute préconception littéraire et à se révéler en d'autres mots.

Les «armes miraculeuses » de Patrick Chamoiseau consistent peut-être dans cette stratégie de dissimulation : il s'agit de montrer que la littérature n'a certainement pas le pouvoir rêvé par les surréalistes d'une libération totale, cependant une avancée basée sur la métamorphose semble tout à fait envisageable.

\section{BIBLIOGRAPHIE}

Bernabe Jean; Chamoiseau Patrick; ConfIANT Raphael (1993), Éloge de la créolité/In praise of creoleness, Paris, Gallimard, 128 p.

CURTIUS Ernst Robert (1998), Evropská literatura a latinský středověk, Praha, Triáda, $740 \mathrm{p}$.

Chamoiseau Patrick (1986), Chronique des sept misères, Paris, Gallimard, coll. «Folio », $281 \mathrm{p}$.

Chamoiseau Patrick (1988), Au temps de l'antan, Paris, Édition Hatier, coll. «Fées et gestes », 107 p.

Chamoiseau Patrick (1988), Solibo Magnifique, Paris, Gallimard, coll. « Folio », $244 \mathrm{p}$.

Chamoiseau Patrick (1992), Texaco, Paris, Gallimard, collection «Folio », $503 \mathrm{p}$.

ChamoISEAU Patrick (1993), Lettre aux traducteurs (inédite) Martinique, Lamentin, le 8 juin 1993.

CHAMOISEAU Patrick (1997), L'esclave vieil homme et le molosse, Paris, Gallimard, coll. « Folio », 149 p.

Chamoiseau Patrick (2013), Veilles et Merveilles créoles. Contes du pays Martinique, Paris, Le Square éditeur, 89 p.

DURAND Gilbert (1996), Champs de l'imaginaire, Grenoble, ELLUG, 263 p. 
GAUVIN Lise (1997), L'écrivain francophone à la croisée des langues, Paris, Éditions Karthala, pp. 35-47.

GLISSANT Edouard (1988), «Un marqueur de paroles » in: Chronique des sept misères, Paris, Gallimard, coll. « Folio », pp. 3-6.

HAZAËL-MASSIEUX Marie-Christine (1998), « Chamoiseau écrit-il en créole ou en français ?» in : Etudes créoles, vol. XXI, pp. 111-126.

JOUBERT Jean-Louis (2006), Les voleurs de langue. Traversée de la francophonie littéraire. Paris, Philippe Rey, $130 \mathrm{p}$.

KUNDERA Milan (2009), Une rencontre. Paris, Gallimard, pp. 99-120.

RALPH Ludwig (1994), Écrire la "parole de nuit». La nouvelle littérature antillaise, Paris, Gallimard, pp. 151-158.

STANZEL Franz K. (1964), Typische Formen des Romans, Göttingen, Vandenhoeck und Ruprecht, 81 p. 